\title{
Risk of incident gastroesophageal reflux disease (GERD) in patients with sleep disorders: A population-based cohort study
}

Yu-Hsien Cheng

National Taiwan University

Pei-En Chen

Cheng Hsin General Hospital

Ching-Wen Chien

Tsinghua University

Tao-Hsin Tung ( $\nabla$ ch2876@gmail.com )

Department of Medical Research and Education, Cheng-Hsin General Hospital

Research article

Keywords: Gastroesophageal reflux disease, sleep disorders, follow-up study

Posted Date: March 30th, 2020

DOI: https://doi.org/10.21203/rs.3.rs-19848/v1

License: (a) (1) This work is licensed under a Creative Commons Attribution 4.0 International License. Read Full License 


\section{Abstract}

Background. Sleep disorders pose a serious threat to human, which may cause variety diseases. Recent reviews on the epidemiology examines that gastroesophageal reflux disease (GERD) have different levels of causality about asthma, chronic dry cough, sleep apnea, non-cardiac chest pain, chest tightness, chronic bronchitis. The relationship between sleep disorders and gastroesophageal reflux disease (GERD) has been explained in various ways, but only limited reports on the complication rates in Taiwan. The objective of this study is to evaluate the risk of incident gastroesophageal reflux disease (GERD) in Taiwanese people with sleep disorders.

Methods. We used the Taiwan's National Health Insurance Research Database to conduct a nationwide population-based cohort study to assess the risk of incident GERD in people with sleep disorders.

Results. A total of 66133 sleep disorders patients and 264,532 non-sleep disorders controls were included. Sleep disorders was a risk factor of incident GERD (adjusted hazard ratio being $1.722,95 \% \mathrm{Cl}$ : $1.69-1.76, p<0.001$ ) after controlling potential confounders including age, gender, and comorbidities.

Conclusions. In conclusion, patients with sleep disorders had a significantly higher incidence of GERD than those without sleep disorders.

\section{Background}

The field of sleep disorders encompasses a broad range of phenomena; such us insomnia, excessive sleepiness, sleep apnea and many other sleep disorders [1]. There are some symptoms about insomnia including insufficient sleep, tossing and turning, feeling tired during the day, and waking up in the middle of night. Some research showed that several factors about reducing sleep quality; such us having a very stressful life [2, 3], unemployed, shift workers [1], or sleeping in an inadequate bedrooms [3] are associated with an increasing risk for sleep disorders in the general population. Sleep disorders is not only increase an individual's obesity and insulin resistance [4-6] and risk for a variety of physical and mental disorders [7], but also decrease one's quality of life, cognitive, emotional, and social functioning and academic performance [8]. Insufficient sleep will pose a serious threat to brain and lead beta-amyloid (one kind of proteins is associated with Alzheimer's disease) accumulation, more and more, and then loss memory $[9,10]$.

The subject symptoms of Gastroesophageal reflux disease (GERD) are mainly heartburn, direct acid injuries such as esophageal erosion, respiratory diseases such as asthma and any other complications including sleep disorders. Recently, there are studies reporting the association of sleep disorders with GERD. GERD is defined as a condition that develops when the reflux of stomach contents causes troublesome symptoms or complications, is a common chronic disorders prevalent in many countries [11]. It is associated with a huge economic burden in the western countries and significantly decreased quality of life [12]. Recent reviews on the epidemiology examines that GERD have different levels of causality about asthma, chronic dry cough, sleep apnea, non-cardiac chest pain, chest tightness, chronic 
bronchitis $[13,14]$. Risk factors in GERD include obesity, pregnancy, smoking, hiatal hernia and specific drugs, including antihistamines, calcium channel blockers, antidepressants and sedatives that medicine help sleep [15]. Some studies even show that chronic life stress and psychological disturbances, such as depression, anxiety, and somatization increase their symptoms $[16,17]$.

The relationship between sleep disorders and GERD has been explained in various ways, and to date there have been only limited reports on the complication rates in Taiwan. We conducted a nationwide population-based study on the incidence of GERD to elucidate the relationship between sleep disorders and GERD in the Taiwanese population.

\section{Methods}

\section{Data source}

The Taiwan National Health Insurance (NHI) Program was enacted in 1995. The National Health Insurance (NHI) program is a mandatory health insurance program offering comprehensive medical care for more than 23 million residents, and enrolling participants exceeded $99 \%$ of the population of Taiwan. The NHIRD contains comprehensive information on clinical visits, including prescription details and the diagnoses recorded are coded according to the International Classification of Disease, Ninth Revision, Clinical Modification (ICD-9-CM) [18-20]. Based on the Personal Information Protection Act, the NHIRD cryptographically assigned an anonymous code to each enrollee for protecting the confidentiality therefore informed consent was waived. Therefore, several previous studies have revealed a high accuracy and validity of the diagnoses in the NHIRD, it is therefore suitable to use the NHIRD to examine the longitudinal association between gastroesophageal reflux disease (GERD) and sleep disorders.

In this study, we used the data set 'Longitudinal Health Insurance Database 2000', comprising data on one million people (approximately $5 \%$ of Taiwan's population), which were systematically and randomly sampled from the LHID2005. The NHIRD has been used extensively in many epidemiologic studies in Taiwan [21].

\section{Study population}

The flowchart about how to select study population and some excluding criteria is illustrated in Figure 1. We excluded the people who had been diagnosed with GERD before 2000 for purpose of avoiding overestimation of the risk of incident GERD. By using ICD-9-CM codes (78050, 78057, and 30740), we identified subjects diagnosed with sleep disorders. In the previous NHIRD studies about sleep disorders, these codes have been used. The exposed group was composed of subjects with sleep disorders newly diagnosed by a psychiatrist since 2000 onwards; conversely, those who were not diagnosed with sleep disorders were considered as the control subjects some of which were made up control group. The index date is the date when the sleep disorders was first diagnosed, while the index date for the control group was January 1 st, 2000. 


\section{Outcomes and potential confounders}

The claim data of the NHIRD is completeness and accuracy, including medication history and ICD9 diagnosis have been validated NHIRD [18-20,22]. The period of GERD subjects follow-up was the time from the index date to the date of the first diagnosis in the inpatient or outpatient records. The censored time of subjects without GERD was from the index date to the end of 2012 or the date to withdrawing the $\mathrm{NHI}$ program. We confirmed the endpoints by requiring records of the diagnosis at least two occasions in 180 days so as to enhance the accuracy of outcomes.

\section{Statistical analysis}

To migrate selection-bias, frequently matching was conducted in which one sleep disorders patient in the cohort was matched four counterparts in the comparison cohort. In order to compare continuous and categorical data, we used the Student's t-test and Chi-squared test respectively. The Kaplan-Meier method was used for survival analysis, with the log-rank test used for comparisons between the sleep disorders and control groups. In addition, the Cox regression model was used to investigate the hazard ratio (HR), between the two groups after adjustment for demographic data (age, sex) and Charlson Comorbidity Index. A two-sided P-value of $<0.05$ was considered statistically significant. All statistical analyses were conducted by using the SAS software version 9.2.

\section{Results}

The basic demographic data of the study population are illustrated in Table 1. During the study period from 2000 to 2012, we identified 66,133 people with sleep disorders and 264,532 controls without sleep disorders. The mean age was the same $(48.09 \pm 16.97)$ for people with and without sleep disorders. Overall, the risk of developing GERD in sleep disorder cohort (incidence rate: 1.21) was 1.29-fold higher than that of the control group (incidence rate: 0.94).

The Kaplan-Meier curve of disease-free probability of GERD stratified by sleep disorders group is illustrated in Figure 2. The GERD-free probability in the sleep disorders group was significantly lower than that in non-sleep disorders controls ( $\mathrm{p}$-value for log-rank test <0.01). Figure 3 shows the sex-specific cumulative survival curves for GERD in sleep disorders vs. non-sleep disorders. The cumulative survival of GERD in the sleep disorders group was significantly lower than that in non-sleep disorders controls both in men and women subgroup ( $p$-value for log-rank test $<0.01$ ). In addition, Figure 4 also indicates that the cumulative survival of GERD in the sleep disorders group was significantly lower than that in nonsleep disorders controls both in $\geqq 50$ years old and $<50$ years old subgroup ( $p$-value for log-rank test $<0.01)$.

The effect of independently associated risk factors upon incident GERD was examined using the multiple Cox regression models. As is depicted in Table 2, subsequent to adjustment for confounding factors, gender (male vs. female, $\mathrm{HR}=0.87,95 \% \mathrm{Cl}: 0.86-0.89)$, age $(\mathrm{HR}=1.01,95 \% \mathrm{Cl}: 1.00-1.02)$, sleep disorders 
(yes vs. no, $\mathrm{HR}=1.72,95 \% \mathrm{Cl}$ 1.69-1.76), and Charlson Comorbidity Index (HR=1.07, 95\%Cl: 1.04-1.12) appeared to be statistically significantly related to incident GERD.

\section{Discussion}

\section{Clinical implications}

This study is the first population-based study conducted to explore the association between sleep disorders and GERD. In the United States and Europe, since around 2000 GERD has been increasingly reported as a complication in patients with obstructive sleep apnea syndrome (OSAS), which one kind of sleep disorders. In addition, at least $50 \%$ of patients with sleep disturbance seen in primary care practices have comorbidity conditions [23]. Specifically, Taylor et al found a higher prevalence of gastrointestinal (GI) problems in those with chronic insomnia compared with those without insomnia (33.6\% vs. 9.2\%; Odds ratio: $3.33,95 \% \mathrm{Cl}: 1.83-6.05)$ [24].

In the past studies, GERD was a risk factor for sleep disorders, and many articles have hypothesized that this association appears to be bidirectional [25-27]. In the existing literature, there is little evidence show the causality between sleep disorders and GERD. Patients with sleep disorders may cause worse sleep quality and indirectly influencing not only mental but also digestive system. Identically, in these two diseases, there are some common risk factors [28]. When the incidence of a disease increases, the other one will naturally increase. Pattern of gastritis in $\mathrm{H}$. pylori infected patients could be association with GERD [29]. Apparently, gastritis is likely to deteriorate GERD, if it is not well controlled. In addition, stress and lifestyle are cardinal risk factors in gastritis, so when the sleep disorders is improved, it may reduce the incidence of gastritis and then reduce GERD indirectly [30]. In clinical trials, the side effects of sedatives which are taken in patients with sleep disorders also increase the incidence of GERD, which could be the reason why increase the incidence of GERD while having medication treatment [31].

\section{Methodological considerations}

Although based on the long-term follow-up study design, that is, being prospective in design as well as in a well defined patient population, we not only determined the cumulative incidence of GERD, but also further explored the prognostic factors related to incident GERD. Nonetheless, it is still important to consider other potential limitations of this study. Firstly, the data source of this study was the NHIRD which lacked relevant variables such as stress indicators, living habits even side effect of medicine. A misclassification bias might have been present. Nevertheless, it seems reasonable to assume that any misclassification bias was not related to incident GERD, thus could be viewed as nondifferential misclassification. Secondly, there might have been some cases of milder GERD that were delayed in diagnosis or were not detected during the follow-up. Thirdly, the level of evidence derived from cohort studies is generally lower than that from randomized trials because of potential biases related to unknown confounders that could not be adjusted. Finally, the effect of treatment on sleep disorders could not be thoroughly studied. Information from clinical treatments beyond insurance coverage, treatment compliance, and treatment duration were not available in the NHIRD 


\section{Conclusion}

In conclusion, this study focused on the casual relationship between sleep disorders and GERD. Our study clarified the relationship between sleep disorders and GERD in the Taiwanese population. However, maybe sleep disorders and GERD is a vicious circle that deserves more research in the future.

\section{Abbreviations}

\section{GERD}

gastroesophageal reflux disease

NHIRD

National Health Insurance Research Database

$\mathrm{NHI}$

National Health Insurance

\section{Declarations}

\section{Ethics approval and consent to participate}

The study is in accordance with Helsinki Declaration. As the National Health Research Institute had addressed the confidentiality assurance issue, a full review of this study was waived by the Institution Review Board of Taipei Veterans General Hospital (IRB-TPEVGH No: 2015-11-001BC).

The data supporting the conclusions of this article are included within the article.

\section{Consent for publication}

Not applicable

\section{Availability of data and material}

All data underlying the findings are within the paper

\section{Competing interests}

The authors have no proprietary interest in any aspect of this study.

\section{Funding}

There was no additional financial support from public or private sources.

\section{Authors' contributions}

YH.C, PE.C, CW.C, and TH.T conducted the study and drafted the manuscript. YH.C and PE.C participated in the design of the study and performed statistical analyses. CW.C, and TH.T conceived the study, and participated in its design and coordination. All of the authors read and approved the final manuscript. 


\section{Acknowledgements}

The authors thank the Sunflower Statistical Consulting Company, Kaohsiung, Taiwan for statistical advice.

\section{References}

[1]Ohayon MM, Smolensky MH, Roth T. Consequences of shiftworking on sleep duration, sleepiness, and sleep attacks. Chronobiol Int 2010;27:575-589.

[2]Ohayon MM, Hong SC. Prevalence of insomnia and associated factors in South Korea. J Psychosom Res 2002;53:593-600.

[3]Ohayon MM, Partinen M. Insomnia and global sleep dissatisfaction in Finland. J Sleep Res 2002;11:339-346.

[4]Chen X, Beydoun MA, Wang Y. Is sleep duration associated with childhood obesity? A systematic review and meta-analysis. Obesity (Silver Spring) 2008;16:265-274.

[5]Fatima Y, Doi SA, Mamun AA. Longitudinal impact of sleep on overweight and obesity in children and adolescents: a systematic review and bias-adjusted meta-analysis. Obes Rev 2015;16:137-149.

[6]Pyykkönen AJ, Isomaa B, Pesonen AK, et al. Sleep duration and insulin resistance in individuals without type 2 diabetes: the PPP-Botnia study. Ann Med 2014;46:324-329.

[7]Ferrie JE, Kumari M, Salo P, et al. Sleep epidemiology--a rapidly growing field.

Int J Epidemiol 2011;40:1431-1437.

[8]Asarnow LD, McGlinchey E, Harvey AG. The effects of bedtime and sleep duration on academic and emotional outcomes in a nationally representative sample of adolescents. $J$ Adolesc Health 2014;54:350-356.

[9]Mayeux R, Stern Y. Epidemiology of Alzheimer disease. Cold Spring Harb Perspect Med 2012;2: pii: a006239.

[10]Musiek ES, Xiong DD, Holtzman DM. Sleep, circadian rhythms, and the pathogenesis of Alzheimer disease. Exp Mol Med 2015;47:e148.

[11]Vakil N, van Zanten SV, Kahrilas $P$, et al. The Montreal definition and classification of gastroesophageal reflux disease: a global evidence-based consensus. Am J Gastroenterol 2006;101:1900-1920. 
[12]Sharma P, Wani S, Romero Y, et al. Racial and geographic issues in gastroesophageal reflux disease. Am J Gastroenterol 2008;103:2669-2680.

[13]Dimache M, Turcan E, Nătase M. Noncardiac chest pain and gastroesophageal reflux disease. Rev Med Chir Soc Med Nat lasi 2010;114:342-348.

[14]Morice AH. Epidemiology of cough. Pulm Pharmacol Ther 2002;15:253-259.

[15]Richter JE, Rubenstein JH. Presentation and Epidemiology of Gastroesophageal Reflux Disease. Gastroenterology 2018;154:267-276.

[16]Naliboff BD, Mayer M, Fass R, et al. The effect of life stress on symptoms of heartburn. Psychosom Med 2004;66:426-434.

[17]Song EM, Jung HK, Jung JM. The association between reflux esophagitis and psychosocial stress. Dig Dis Sci 2013;58:471-477.

[18]Cheng CL, Kao YH, Lin SJ, et al. Validation of the National Health Insurance Research Database with ischemic stroke cases in Taiwan. Pharmacoepidemiol Drug Saf 2011;20:236-242.

[19]Cheng $\mathrm{CL}$, Lee $\mathrm{CH}$, Chen PS, et al. Validation of acute myocardial infarction cases in the national health insurance research database in Taiwan. J Epidemiol 2014;24:500-507.

[20]Cheng CL, Chien HC, Lee CH, et al Validity of in-hospital mortality data among patients with acute myocardial infarction or stroke in National Health Insurance Research Database in Taiwan. Int J Cardiol 2015;201:96-101.

[21]National Health Research Institutes. Longitudinal Health Insurance Databases. Accessed on June 18 2019. Available at: https://nhird.nhri.org.tw/en/Data_Subsets.html\#S3.

[22]Wu CS, Lai MS, Gau SS, et al. Concordance between patient self-reports and claims data on clinical diagnoses, medication use, and health system utilization in Taiwan. PLoS One 2014;9:e112257.

[23]Roth T, Roehrs T. Insomnia: epidemiology, characteristics, and consequences. Clin Cornerstone 2003;5:5-15.

[24]Taylor DJ, Mallory LJ, Lichstein KL, et al. Comorbidity of chronic insomnia with medical problems. Sleep 2007;30:213-218.

[25]Jung HK, Choung RS, Talley NJ. Gastroesophageal reflux disease and sleep disorders: evidence for a causal link and therapeutic implications. J 
Neurogastroenterol Motil 2010;16:22-29.

[26]Kusano M, Kouzu T, Kawano T, et al. Nationwide epidemiological study on gastroesophageal reflux disease and sleep disorders in the Japanese population. J Gastroenterol 2008;43:833-841.

[27]Lindam A, Ness-Jensen E, Jansson C, et al. Gastroesophageal Reflux and Sleep Disturbances:

A Bidirectional Association in a Population-Based Cohort Study, The HUNT Study. Sleep 2016;39:14211427.

[28]McDonald-Haile J, Bradley LA, Bailey MA, et al. Relaxation training reduces symptom reports and acid exposure in patients with gastroesophageal reflux disease.

Gastroenterology 1994;107:61-69.

[29] Nobakht H, Boghratian A, Sohrabi M, et al. Association between Pattern of Gastritis and Gastroesophageal Reflux Disease in Patients with Helicobacter Pylori Infection. Middle East J Dig Dis 2016;8:206-211.

[30]Carabotti M, Esposito G, Lahner E, et al. Gastroesophageal reflux symptoms and microscopic esophagitis in a cohort of consecutive patients affected by atrophic body gastritis: a pilot study. Scand J Gastroenterol 2019;54:35-40.

[31] Fass R, Quan SF, O'Connor GT, et al. Predictors of heartburn during sleep in a large prospective cohort study. Chest 2005;127:1658-1666.

\section{Tables}

Table1 Basic demographic data and gastroesophageal reflux disease outcome for patients with and without sleep disorders (Both case group and control group are revealed; SD: standardized difference; N: number) 


\begin{tabular}{|c|c|c|c|c|c|}
\hline & \multirow{2}{*}{$\begin{array}{c}\text { Total } \\
\mathrm{N}(\%) \text { or }\end{array}$} & \multicolumn{2}{|c|}{ With sleep disorders } & \multicolumn{2}{|c|}{ Without sleep disorders } \\
\hline & & $\mathrm{N}(\%)$ or & Incidence Rate & $\mathrm{N}(\%)$ or & Incidence Rate \\
\hline & Mean \pm SD & Mean \pm SD & $(95 \% \mathrm{CI})$ & Mean \pm SD & $(95 \% \mathrm{CI})$ \\
\hline & 330,665 & 66,133 & $1.21(1.15-1.3)$ & 264,532 & $0.94(0.87-1.00)$ \\
\hline & $130,550(39.5 \%)$ & $26,110(39.5 \%)$ & $1.05(0.99-1.21)$ & $10,440(39.5 \%)$ & $0.78(0.65-1.08)$ \\
\hline & $200,115(60.5 \%)$ & $40,023(60.5 \%)$ & $1.42(1.37-1.67)$ & $160,092(60.5 \%)$ & $1.11(1.05-1.20)$ \\
\hline & $48.09 \pm 16.97$ & $48.09 \pm 16.97$ & $1.23(1.1-1.35)$ & $48.09 \pm 16.97$ & $1.06(1.01-1.34)$ \\
\hline norbidity & $2.74 \pm 2.4$ & $2.74 \pm 2.4$ & $1.21(1.17-1.6)$ & $2.74 \pm 2.4$ & $1.02(0.93-1.18)$ \\
\hline
\end{tabular}

Table 2 Cox regression of the risk factors related to gastroesophageal reflux disease

\begin{tabular}{|c|c|c|c|c|c|}
\hline \multicolumn{6}{|c|}{ Gastroesophageal reflux disease } \\
\hline \multicolumn{6}{|c|}{ (yes vs. no) } \\
\hline Variables & $\beta$ & $\mathrm{SE}$ & Hazard ratio & $95 \%$ confidence interval & $\mathrm{p}$-value \\
\hline age & 0.009 & 0.01 & 1.01 & $1.00-1.02$ & $<0.001$ \\
\hline Gender (male vs. female) & -0.14 & 0.01 & 0.87 & $0.86-0.89$ & $<0.001$ \\
\hline Sleep disorders (yes vs. no) & 0.54 & 0.01 & 1.72 & $1.69-1.76$ & $<0.001$ \\
\hline Charlson Comorbidity Index & 0.77 & 0.18 & 1.07 & $1.04-1.12$ & 0.18 \\
\hline
\end{tabular}




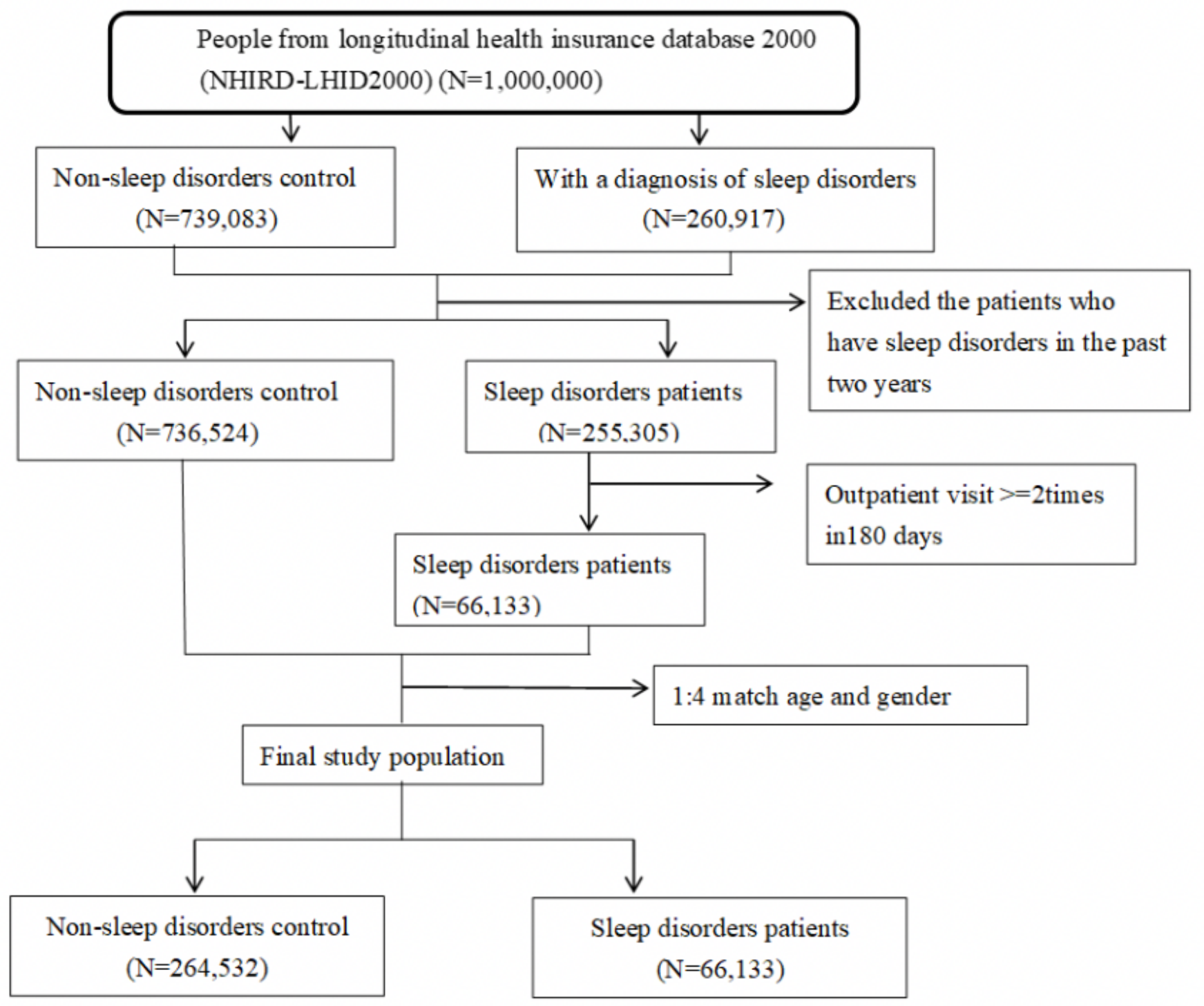

Figure 1

Flow chart of selection of the study population 


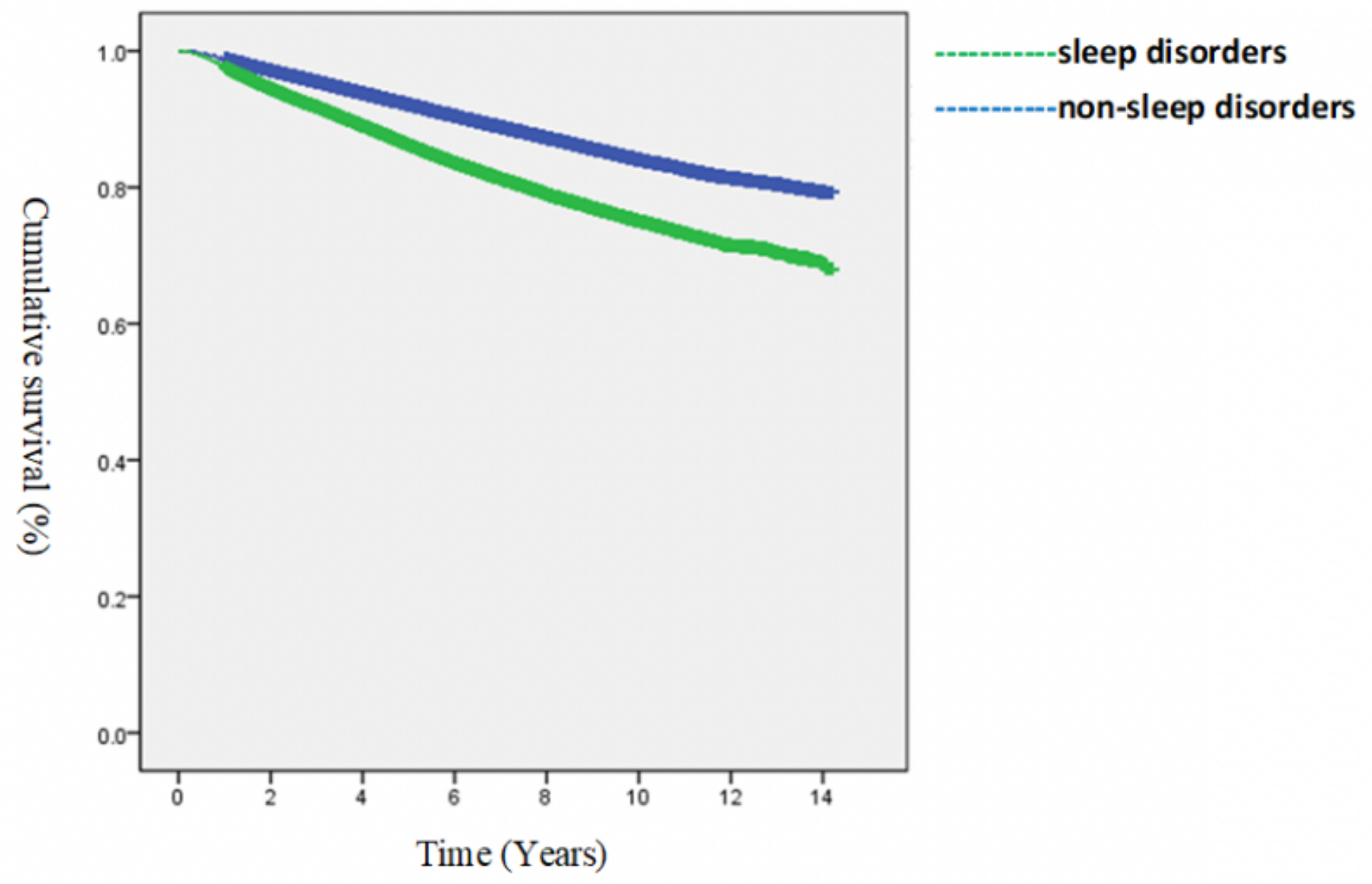

Figure 2

Cumulative survival curves for GERD in sleep disorders vs. non-sleep disorders 
(a) men

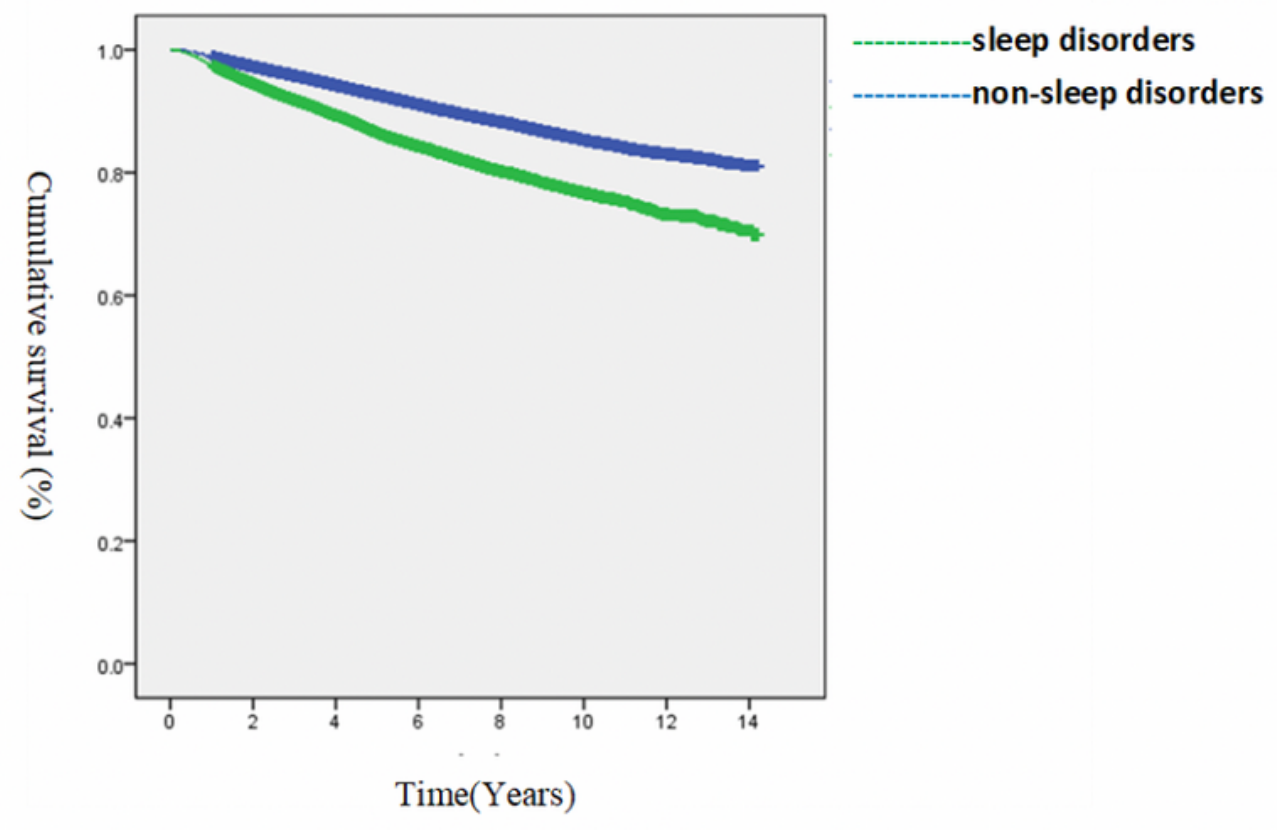

(b) women

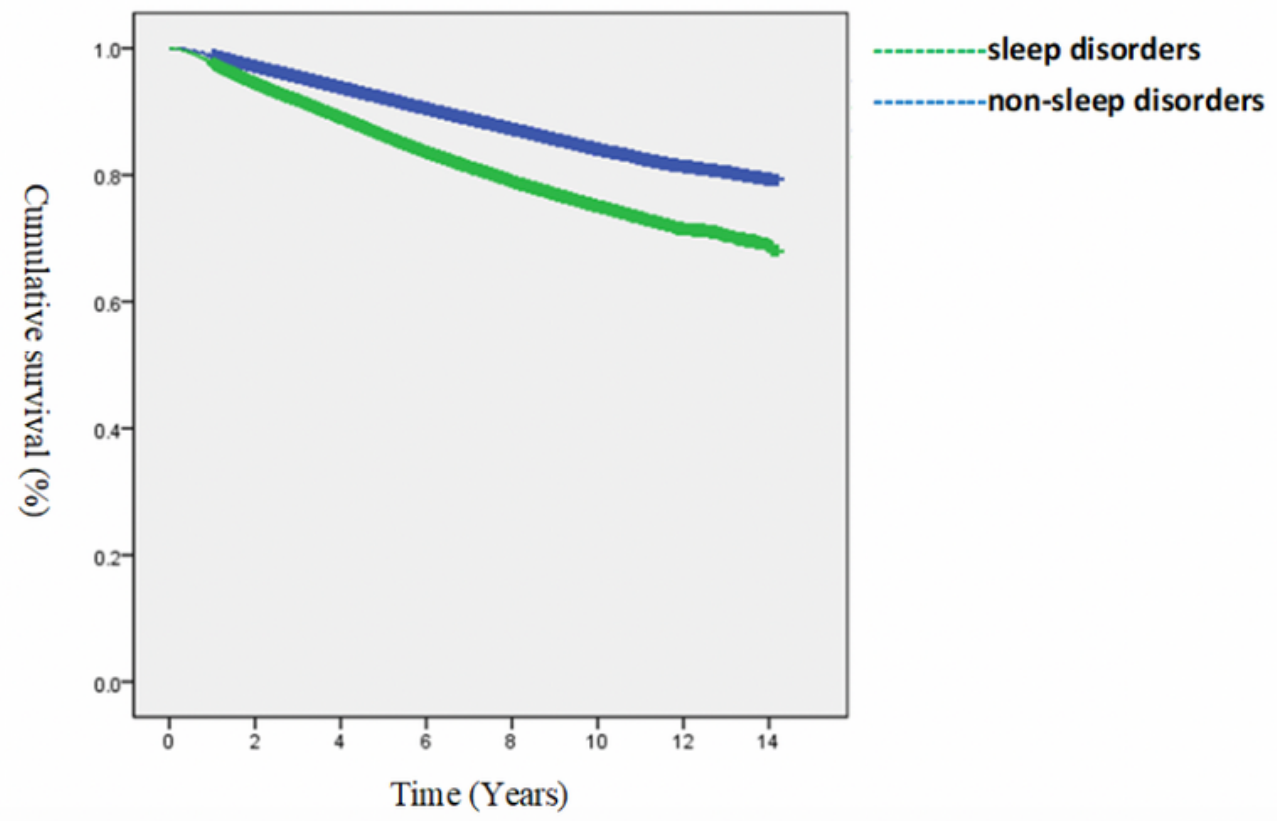

\section{Figure 3}

Sex-specific cumulative survival curves for GERD in sleep disorders vs. non-sleep disorders (a) men (b) women 
(a) $<50$ years old
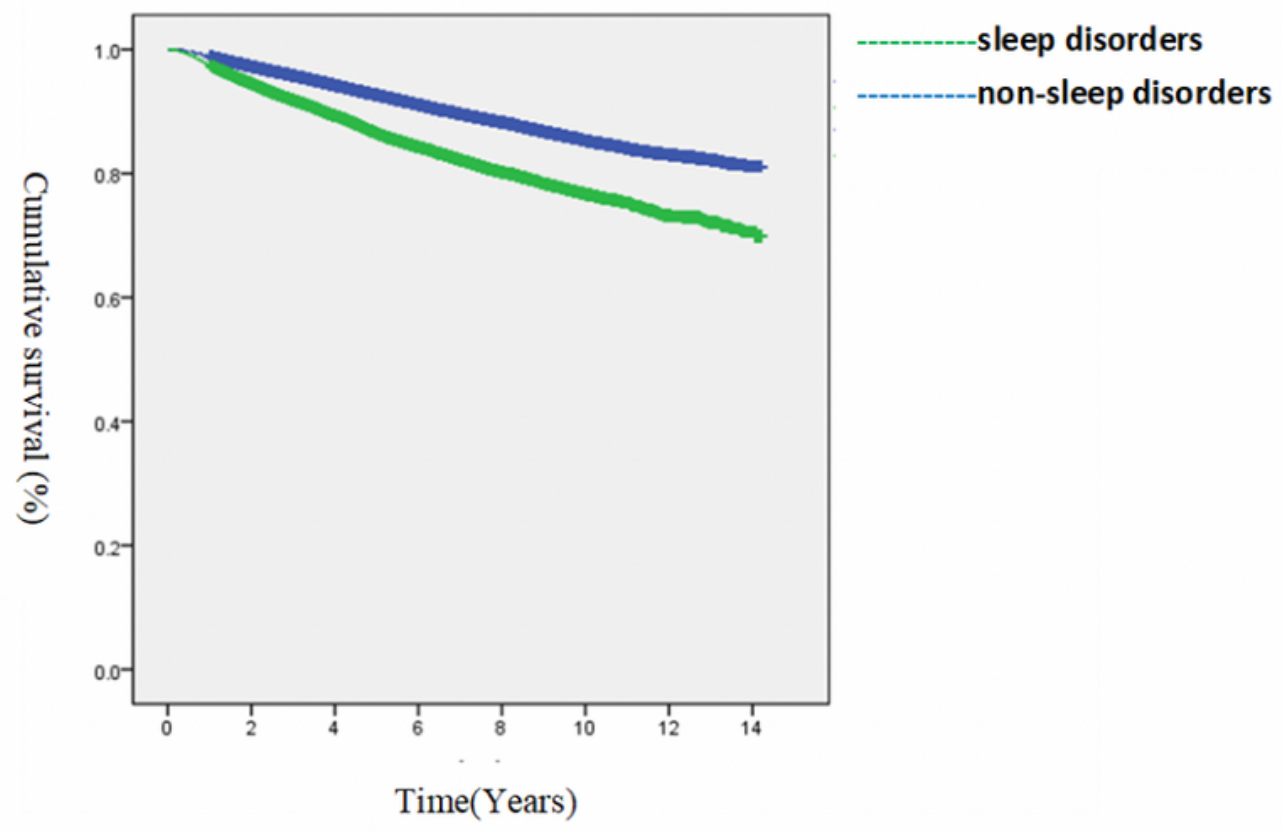

(b) $\geqq 50$ years old

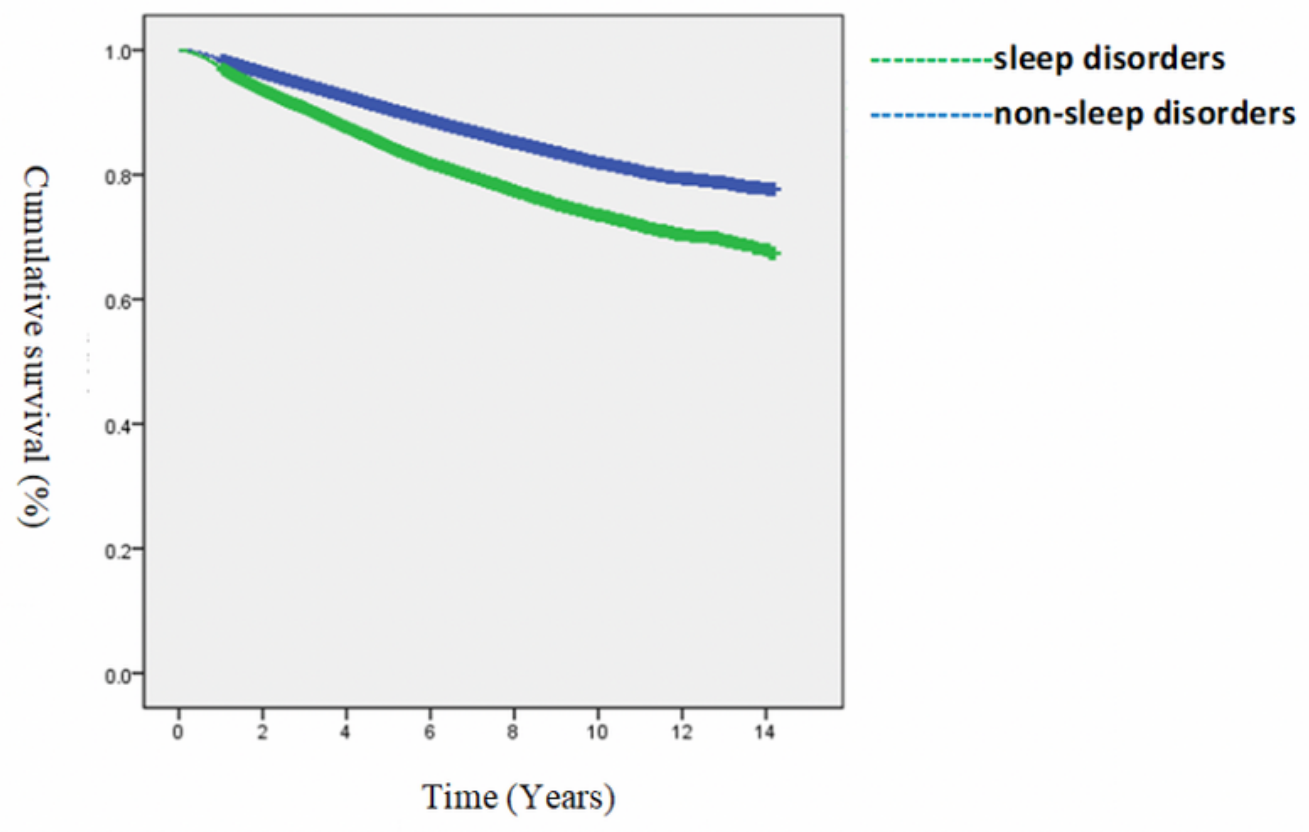

\section{Figure 4}

Age-specific cumulative survival curves for GERD in sleep disorders vs. non-sleep disorders (a) $<50$ years old $(b) \geqq 50$ years old 